\title{
A Design of Full-automatic Advanced Sludge Dewatering System
}

\author{
Xuxin Zhao ${ }^{1, \text { a }}$, Huide $\mathrm{Fu}^{2, \mathrm{~b}}$, Lin Fang ${ }^{3, \mathrm{c}}$ and Lei $\mathrm{Ma}^{4, \mathrm{~d}}$ \\ 1,2,3.4 College of Chemical and Environmental Engineering, Shenzhen University, Shenzhen, \\ 518060, China \\ azhaoxx@szu.edu.cn, ${ }^{b}$ huidef@szu.edu.cn, ${ }^{\mathrm{c}}$ fanglin@szu.edu.cn, ${ }^{\mathrm{d}}$ leima@szu.edu.cn
}

Keywords: Advanced sludge dewatering; Sludge conditioning; mechanically dewatering; Auto-control

Abstract: Advanced Sludge dewatering system is investigated by the approaches of physical and chemical pretreatment process to improve sludge dewatering performance, based on the degree of difficulty or ease together with the relationship analysis of the existing form of the sludge water. Systems of sludge pre-treatment, sludge filter press and auto-control are elucidated significantly, with the aim that accomplishing the sludge deep dewatering, and making the moisture content of sludge below 55\%. This approach can suit to the manipulating locally of the sludge in the sewage treatment plant and save the investment of sludge storage storehouse as well as the cost of transportation.

\section{Introduction}

Nowadays, the main disposal methods of sludge may include recycling as fertilizer, landfills, dumping into the sea, incineration and other conversion technologies. Advanced Sludge deep dewatering has become the bottleneck of these disposal technologies, which has been extensively investigated by domestic and foreign scholars $[1,2]$. Most of the dry technologies are hot drying, and mechanical press drying, solar drying, solidification drying, etc. However, due to their own restrictions, they are difficult to meet the actual demand of the sludge deep dehydration. Based on the existing form and characteristics of water in sludge, this paper proposed a technical scheme of deep dewatering of sludge. The dewatering performance of sludge is improved by the approaches of physical and chemical pretreatment process of the sludge, and the water content of the sludge is reduced to below $55 \%$ by using hydraulic press dewatering technology.

\section{Forms and characteristic of water in sludge}

Generally, the water in the sludge can be divided into 2 categories: free water and bound water. Free water can be understood as the free flow of water in the sludge and is not affected by the impact of the solid particles; the bound water is that affected by the property of solid particles. However, dividing the water in the sludge only into 2 categories can not meet the understanding of the sludge water and sludge dewatering performance. Therefore many scholars put forward a more detailed classification of the water in the sludge, which is divided into 4 forms: the void water (about 20\%), the capillary water (about 70\%), the adsorption water (about 7\%), the inner water (about 3\%), as shown in figure 1. The void water is not directly combined with the solid particles, and it is easy to be separated, which is the main object of the sludge concentration. Due to the adhesion of the liquid and the adhesion of the liquid solid surface, the separation of capillary adsorption water formed by the capillary phenomenon requires high mechanical action and more energy. Adsorption water is adsorbed on the surface of the sludge particles, and the sludge is often in a state of colloidal particles. The removal of surface adsorption water requires higher energy. Due to the tight binding of inner water and solid particles, it is difficult to be removed by using mechanical methods. The strength of the combination of water and sludge solid particles from the large to small order is roughly as follows: innerl water, adsorption water, capillary water, pore water. In addition, sludge dewatering is also impacted by the grain size and content of organic matter. 


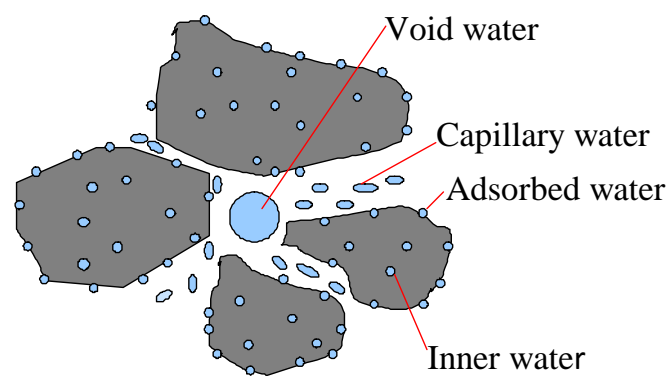

Figure 1. The classification of the water in the sludge

\section{Approach of sludge deep dewatering}

Based on the difficulty level of sludge dewatering and the analysis of water form relations in the sludge, the traditional mechanical dehydration can only remove most of the void water and capillary water, to further reduce the water content of sludge, the Physics and Chemistry Characteristic of sludge must be changed to reduce hydrophilic colloid particles and microbes in the sludge of wall breaking treatment [3]. To solve this problem, we designed a sludge deep dewatering process flow as shown in Fig. 2.

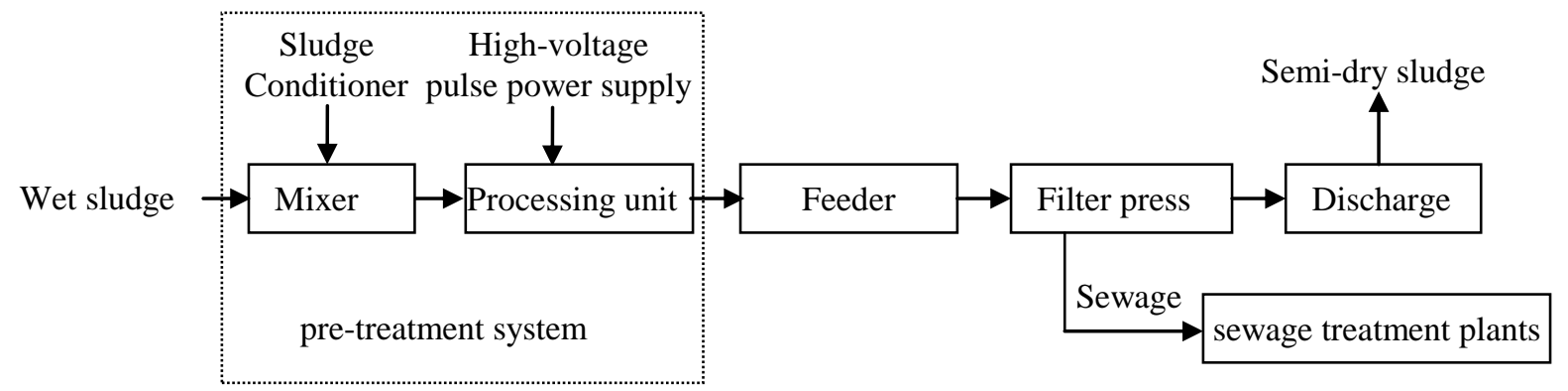

Figure 2. Sludge deep dewatering process flow

System of sludge pre-treatment. The sludge pre-treating system consists of chemical conditioning and physical conditioning subsystems. In the chemical conditioning, based on the analysis of the properties of the matrix of the sludge, by using amine, polymerization alumina, natural polymer modified conditioners composite processing technology, water channel is formed in the sludge internal skeleton structure; in the physical conditioning, through an external high voltage pulse field, the direct action of the electric field and the microbial cell membranes to break the cell wall [4, 5]. The high voltage pulse pretreatment device is shown in Fig. 3.

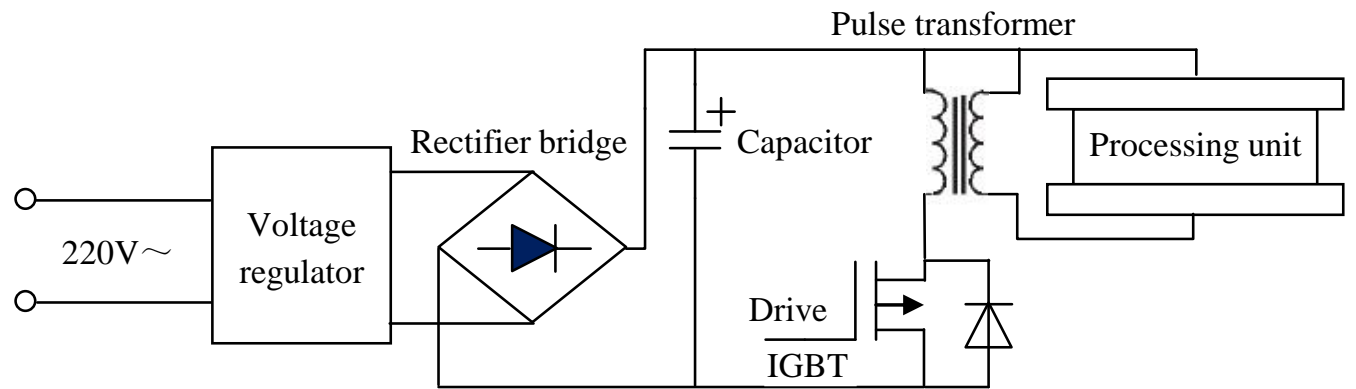

Figure 3. The high voltage pulse pretreatment device

System of sludge filter press. The water content of sewage sludge often is $90 \%$ through the thickening. In order to realize sludge deep dewatering, further dewatering process must be needed. 
The automatic sludge dewatering device was developed [6]. The device integrates the sludge conditioning and filter press techniques. The sludge specific resistance was reduced through the addition of sludge conditioning. The pressure difference produced from the hydraulic pressure between the two sides of the specially-made structure was used as the driving force, which is 4-8 times of the pressure from the conventional dewatering device. With the presence of the sludge conditioning and driving force, the sludge was conditioned and forced to pass through the filtration media. As a result, the filtrate was derived and the filter cake was formed, reaching the aim of extensive dewatering. The sludge after the dewatering through the developed device holds the moisture content lower than 55\%. The sludge filter press developed is shown in Fig. 4.

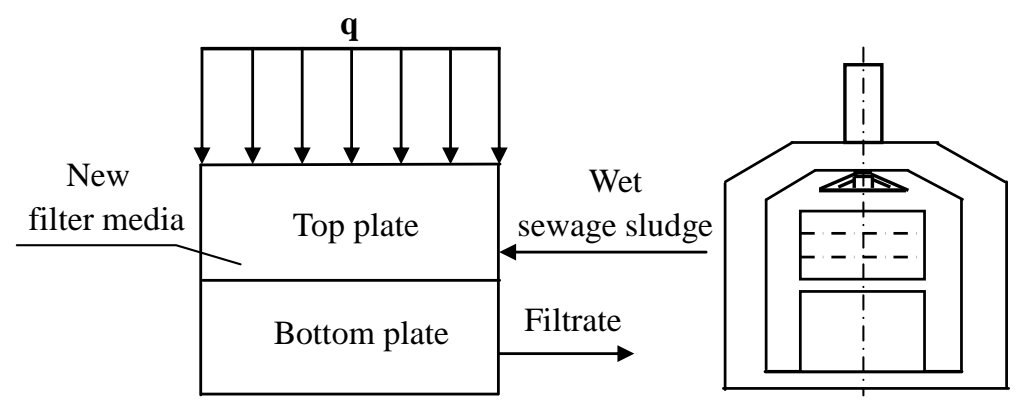

\section{4-a Filter module 4-b Filter press}

Figure.4 Schematic diagram of sludge filter press

Design of auto-control system. For deeply realizing the cooperation of dewatering equipment and pretreatment system to achieve the goal of the sludge deep dewatering, the control system adopts PLC system to control the whole operation process, regulates the process parameters and equipment operation monitoring with touch-screen, as well as achieves the automatic operation of presupposition of the condition parameters, conditioner added, feeding, discharging, filter, etc.

\section{Conclusions}

(1) Sludge deep dewatering system is investigated by the approach of physical and chemical pretreatment to improve sludge dewatering performance, based on the degree of difficulty or ease together with the relationship analysis of forms of the water in sludge.

(2) The design of sludge dewatering drying system adopts vertical structure and modular design, due to the small area of equipments and little energy consumption, easily applied in the sewage treatment plant and thus the investment of sludge storage storehouse is saved together with the cost of transportation. The the sewage treatment plant deals with the sludge completely independently, without any restriction and influence of external conditions, environment, and climate factors. System equipment is easily operated and maintenanced, to yearly keep the continuous and stable operation.

(3) Achieving automatic operation for each station, PLC system controling the whole process of operation is adopted to deeply realize the efficient coordination of dewatering equipment and pretreatment system, for the aim that accomplishing the sludge deep dewatering, and making the moisture content of sludge below $55 \%$. 


\section{Acknowledgements}

This work was financially supported by Shenzhen Research Foundation of Science \& Technology (JCYJ20130329113322731).

\section{References}

[1] J.Q. Xu, W. Cheng and Z. Geng: Application of the diaphragm tupe plate and frame filter process on advanced sludge dewatering, China Water \& Wastewater, Vol. 39, 3(2013), p.87-90.

[2] X.Y. Ji, Y.L. Wang and J. Feng: Study on Dewatering of Activated Sludge Under Applied Electric Field, Environmental Science, Vol.33, 12(2012), p.4393-4399.

[3] F. Lauren, L. Stone and P. Scanlan: Energy Efficiency for Biosolids Treatment: An Update of Best Practices and Case Studies, Proceedings of the Water Environment Federation, Vol.18, (2010), p.847-864.

[4] T. Li, Y.W. Wang, Z.L. Zhao and L.H. You: Design of sewage treatment system based on high-voltage pulsed electric field technology, Electronic Design Engineering, Vol.21, 4(2013), p.104-109.

[5] G.M. Yan, Z.L. Zhao, J.Z. Hou and J.L. Chen: Development of High Energy Pulse Electrical Source and Its Application to Industrial Wastewater Treatment, High Voltage Apparatus, Vol.43, 6(2007), p.466-468.

[6] X.K. Wang, Y. Zhu, Y.N. Zou , LI Jing, Liu Feng etal, Discussion on factors affecting advanced mechanical sludge dewatering, China Water \& Wastewater, Vol.28, 10(2012), p.23-25. 